\title{
6. Diversity of the flora and vegetation of abandoned farmlands
}

\subsection{Diversity of species and habitat groups of the flora}

\author{
Jolanta Adamczyk
}

One hundred and thirty-nine taxa of vascular plants and four taxa of mosses were recorded in the analysed abandoned lands (Tab. 6.1).

In the flora inhabiting abandoned farmlands, there are taxa which prefer various habitat types. The majority of identified species are associated with habitats of dry grasslands or they grow there frequently (24 species). They include Carlina vulgaris, Carex leporina, Centaurea scabiosa, C. stoebe and Thymus pulegioides. Almost equally numerous were groups of species associated with or preferring farmland habitats (22 species), such as Apera spica-venti, Arnoseris minima, Centaurea cyanus, Papaver argemone and species which mostly inhabit ruderal locations, including species of the Rudero-Secalietea class (20 species), e.g. Cirsium arvense, Convolvulus arvensis, Elymus repens and Rumex crispus. The group associated with meadow habitats of the Molinio-Arrhenat- heretea class (19 species) is also numerously represented. They include: Achillea millefolium, Anthoxanthum odoratum, Crepis biennis, Galium mollugo, Rumex acetosa, Stellaria graminea, Trifolium repens and Vicia cracca. Fewer species are found in the group of plants which occur predominantly in forest communities (13 species). They mainly include trees: Pinus sylvestris, Betula pendula, Quercus robur and a few species of herbaceous plants, such as Anthriscus sylvestris and Pteridium aquilinum (Tab. 6.1).

Invasive species of non-native origin should be treated as a separate group. There are 7 of them, which is about $5 \%$ of all the identified plant taxa. They include three species of high invasiveness category (Tab. 6.2). The remaining 34 taxa are cosmopolitan plants, which occur in various types of plant communities.

Table 6.2. Invasive alien plant species recorded on abandoned farmlands of the Łódź Voivodeship

\begin{tabular}{|l|l|l|c|}
\hline \multicolumn{1}{|c|}{ Latin name } & \multicolumn{1}{c|}{ Life form } & \multicolumn{1}{c|}{ Origin } & $\begin{array}{c}\text { Category of } \\
\text { invasiveness }\end{array}$ \\
\hline Anthoxanthum aristatum Boiss. & annual plant & South Europe & I \\
\hline Conyza canadensis (L.) Cronquist & annual plant & North America & I \\
\hline Galinsoga parviflora Cav. & annual plant & North and Central America & I \\
\hline Padus serotina (Ehrh.) Borkh. & tree & North and Central America & IV \\
\hline Quercus rubra L. & tree & North America & IV \\
\hline Setaria viridis (L.) P. Beauv. & annual plant & $\begin{array}{l}\text { South Europe, South-West Asia, } \\
\text { North Africa }\end{array}$ & I \\
\hline Solidago canadensis L. & perennial & North America & IV \\
\hline
\end{tabular}

Categories of invasiveness according to Tokarska-Guzik et al. 2012:

Category I - segetal and ruderal weeds that show high relative abundance, or potentially invasive species that currently occupy a small area;

Category II - species that already show invasive behaviour in some regions, and are increasing the occupied area or the number of localities;

Category III - species occurring at a few localities with high relative abundance or found scattered around many localities;

Category IV - species whose occurrence is very important in Poland - a large number of localities and large populations in patches are known.

Source: own elaboration. 
Apart from Helichrysum arenaria, no protected species or species that are endangered in Poland or the Łódź region were found in the flora of the analysed abandoned lands. Despite that, some of the identified species are not common in the
Łódź Voivodeship, due to their occurrence in rare or endangered phytocoenoses. Such species as Dianthus carthusianorum, Knautia arvensis and Thymus pulegioides are worth mentioning here.

\subsection{Vegetation structure}

\section{Jolanta Adamczyk}

In the analysed abandoned lands, three main structural groups of plant communities (Fig. 6.1) were distinguished using Ward's method of hierarchical cluster analysis. In addition, the use of the IndVal index made it possible to determine the preferences of plant species for individual community group (Tab.6.3). Each community group represents a particular development sta- ge but sometimes, in the same cluster, there are communities typical of the given development stage and communities which are a transition to another stage or community with a different structure. The isolated clusters and analysis of similarities between the plots they were assigned, allowed to identify 8 plant communities with different structures.

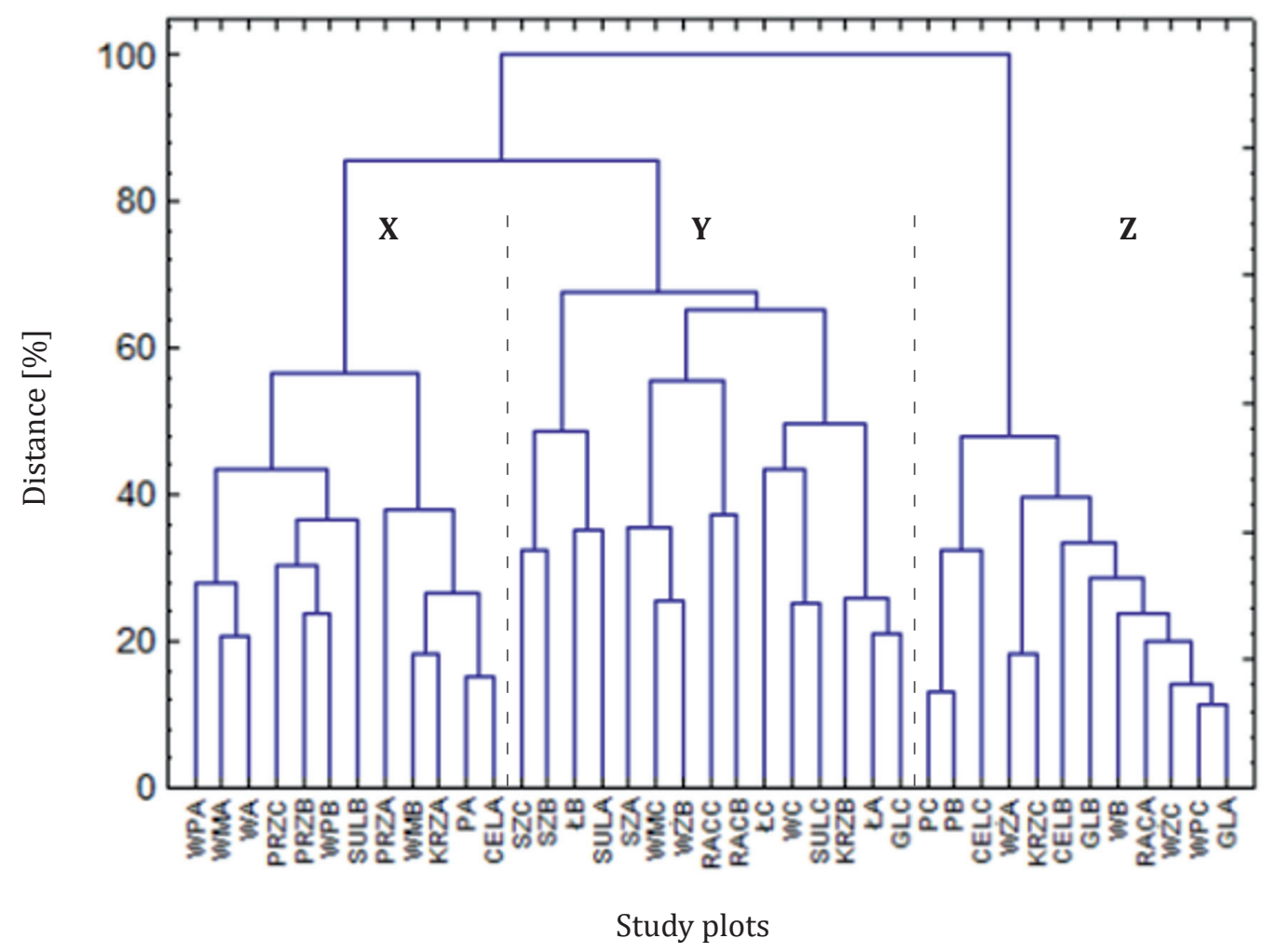

Abbreviations: CELA, CELB, CELC - Celestynów A, Celestynów B, Celestynów C; GLA, GLB, GLC - Glinnik A, Glinnik B, Glinnik C; KRZA, KRZB, KRZC - Krzętle A, Krzętle B, Krzętle C; ŁA, tB, tC - Łagiewniki A, Łagiewniki B, Łagiewniki C; PA, PB, PC - Polesie A, Polesie B, Polesie C; PRZA, PRZB, PRZC - Piskorzeniec A, Piskorzeniec B, Piskorzeniec C; RACA, RACB, RACC - Raciszyn A, Raciszyn B, Raciszyn C; SULA, SULB, SULC - Sulejów A, Sulejów B, Sulejów C; SZA, SZB, SZC - Szymaniszki A, Szymaniszki B, Szymaniszki C; WA, WB, WC - Weronika A, Weronika B, Weronika C; WMA, WMB, WMC - Wola Makowska A, Wola Makowska B, Wola Makowska C; WPA, WPB, WPC - Wola Pszczółecka A, Wola Pszczółecka B, Wola Pszczółecka C; WŻA, WŻB, WŻC - Wola Życińska A, Wola Życińska B, Wola Życińska C

Fig. 6.1. Classification of study plots (hierarchical cluster analysis, Euclidean distance, Ward's method) 
Achillea millefolium $L$

\section{Agrimonia eupatoria L.}

Agrostis capillaris $L$.

Agrostis gigantea ROTH

Anagallis arvensis $L$.

Anthoxanthum aristatum BOISS.

Anthoxanthum odoratum $L$

Anthriscus sylvestris (L.) HOFFM

Apera spica-venti (L.) P. BEAUV.

Arnoseris minima (L.) SCHWEIGG. \& KÖRTE

Arrhenatherum elatius (L) P. BEAUV ex J PRESL \& C PRES

Artemisia absinthium $L$

Artemisia campestris $L$

Artemisia vulgaris

Betula pendula ROTH

Brym sp.

Calamagrostis epigejos (L.) ROTH

Carex hirta $L$.

Carex leporina $L$.

Carlina vulgaris $L$.

Centaurea cyanus $L$

Centaurea scabiosa $L$

Centaurea stoebe $L$.

Cerastium arvense L. s. S.

Ceratodon purpureus (Hedw.) Brid.

Chamaenerion angustifolium (L.) SCOP

Chamomilla recutita (L.) RAUSCHERT

Chenopodium album $L$

Cirsium arvense (L.) SCOP

Cladonia strepsillisf. coralloides Vain

Cladonia furcata (Huds.) Schrad.

Cladonia rangiferina (L.) Weber ex F.H. Wigs

Cladonia arbuscula (Wallr.) Flot.

Cladonia uncialis Elenkin \& Savicz

Convolvulus arvensis $L$.

Conyza canadensis (L.) CRONQUIST

Coronilla varia $L$.

Corynephorus canescens (L.) P. BEAUV

Crataegus monogyna JACQ.

Crepis biennis $L$.

Crepis tectorum $L$. \begin{tabular}{lllllllllllllllllllllllllllllllllllllllllllll}
0,5 & 0,5 & 0 & 0 & 0,5 & 2 & 1 & 0 & 0,5 & 0 & 1 & 0 & 0,5 & 3 & 5 & 0,5 & 3 & 0 & 0,5 & 0 & 0,5 & 0 & 0 & 0 & 2 & 0 & 0,5 & 1 & 0 & 0 & 0 & 3 & 1 & 5 & 0 & 0 & 0 & 0 & 0 \\
\hline
\end{tabular}

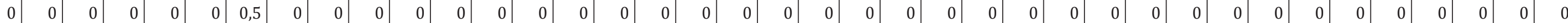

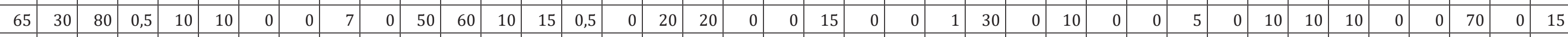

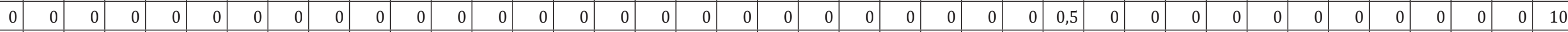

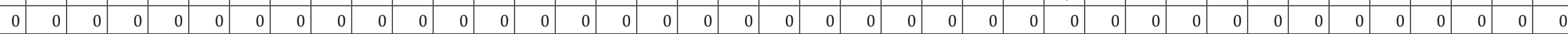
\begin{tabular}{llllllllllllllllllllllllllllllllllllllllll}
0 & 0 & 0 & 0 & 0 & 0 & 0 & 0 & 0 & 1 & 0 & 0 & 0 & 0 & 0 & 0,5 & 0 & 0 & 0,5 & 0 & 0 & 0 & 3 & 0 & 0 & 0 & 0 & 0 & 0,5 & 0 & 0 & 0 & 0 & 0 & 0 & 0 & 0 & 0 & 0 \\
\hline
\end{tabular}

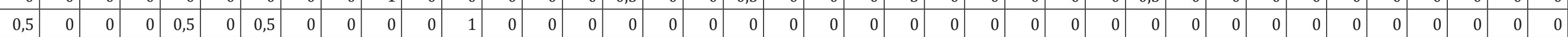

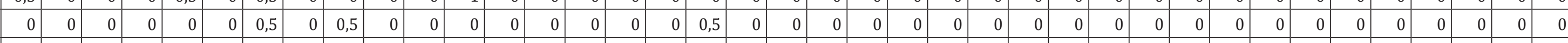

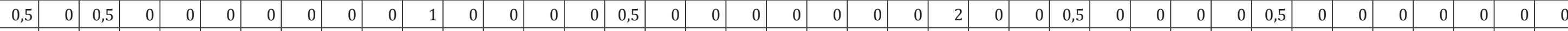
\begin{tabular}{llllllllllllllllllllllllllllllllllllllllllll}
0 & 0 & 0 & 0 & 0 & 0 & 0 & 0 & 0 & 0 & 0 & 0 & 0 & 0 & 0 & 0 & 0 & 0 & 0 & 0 & 0 & 1 & 0 & 1 & 0 & 0 & 0 & 0 & 0 & 0 & 0 & 0 & 0 & 0 & 0 & 0 & 0 & 0 & 0 \\
\hline
\end{tabular}

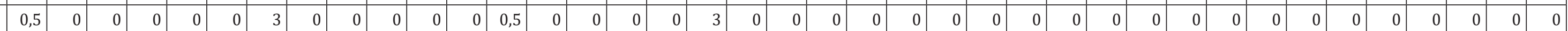

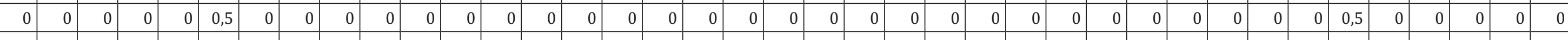

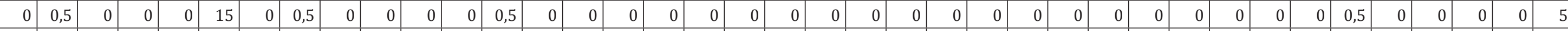

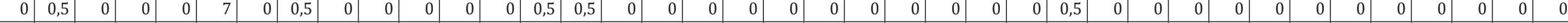

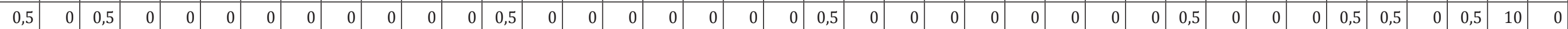

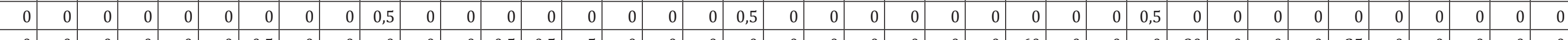

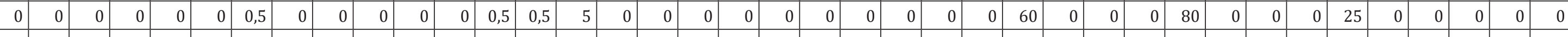

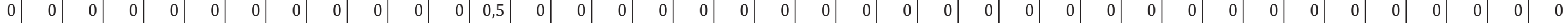
\begin{tabular}{llllllllllllllllllllllllllllllllllllllllllllll}
0 & 0 & 0 & 0 & 0 & 0 & 0 & 0 & 0 & 0 & 0 & 0 & 0 & 0 & 0 & 0 & 0 & 0 & 0 & 0 & 0 & 0 & 0 & 2 & 0 & 0 & 0 & 0 & 0 & 0 & 0 & 0 & 0 & 0 & 0 & 0 & 0 & 0 & 0 \\
\hline
\end{tabular}

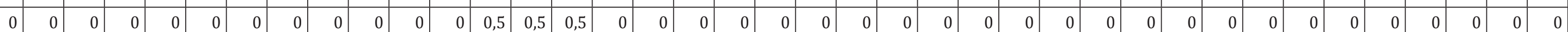

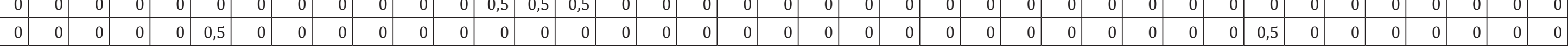
\begin{tabular}{llllllllllllllllllllllllllllllllllllllllll}
0 & 0 & 0 & 0 & 0 & 0 & 0 & 0 & 0 & 0 & 0 & 0 & 0 & 0 & 0,5 & 0 & 0 & 0 & 0 & 0 & 0 & 0 & 0 & 0 & 0 & 0 & 0 & 0 & 0 & 0 & 0 & 0 & 0 & 0 & 0 & 0 & 0 & 0 & 0 \\
\hline
\end{tabular}

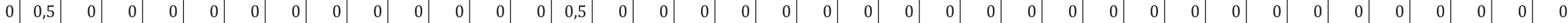

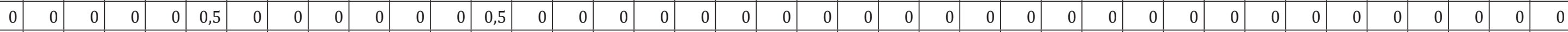

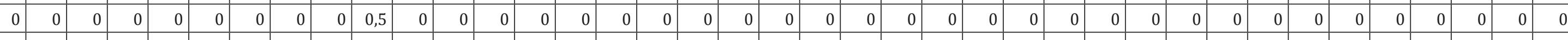

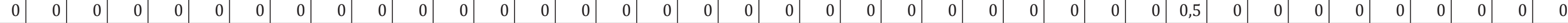
\begin{tabular}{lllllllllllllllllllllllllllllllllllllllll|}
0 & 0 & 0 & 0 & 0 & 0 & 0 & 0 & 0 & 0 & 0 & 0 & 0 & 0 & 0 & 0 & 0 & 0 & 0 & 0 & 0 & 0 & 0 & 0 & 0 & 0 & 0 & 0 & 0 & 0 & 0 & 0,5 & 0 & 0 & 0 & 0 & 0 & 0 & 0 \\
\hline
\end{tabular}

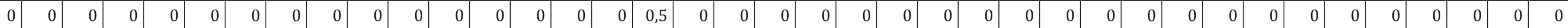

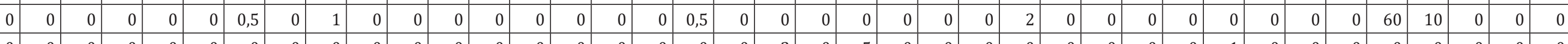

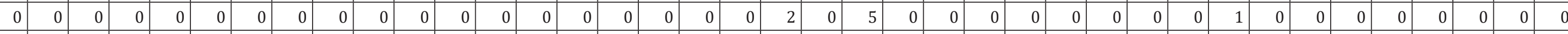

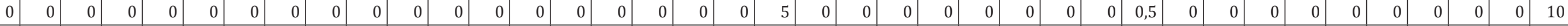
\begin{tabular}{l|l|l|l|l|l|l|l|l|l|l|l|l|l|l|l|l|l|l|l|l|l|l|l|l|l|l|l|l|l|l|l|l|l|l|l|l|l|l}
0 & 0 & 0 & 0 & 0 & 0 & 0 & 0 & 0 & 0 & 0 & 0 & 0 & 0 & 0 & 0 & 0 & 0 & 0 & 0 & 0 & 10 & 0 & 0 & 0 & 0 & 0 & 0 & 0 & 0 & 0 & 0 & 0 & 0 & 0 & 0 & 0 & 0 & 0 \\
\hline
\end{tabular}

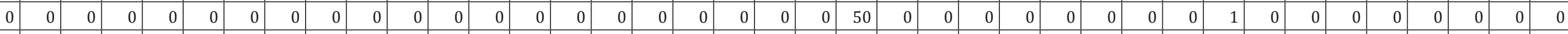

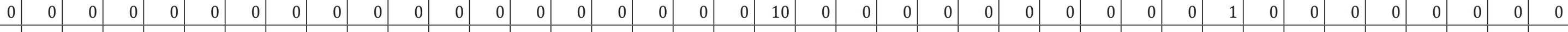
\begin{tabular}{lllllllllllllllllllllllllllllllllllllllllll}
0 & 2 & 0 & 0 & 0 & 0,5 & 0 & 0 & 0 & 10 & 0,5 & 0 & 0 & 0,5 & 0,5 & 0,5 & 0,5 & 0 & 3 & 0 & 0 & 0 & 0 & 0 & 1 & 0 & 0,5 & 0,5 & 0,5 & 0 & 0 & 0,5 & 0 & 0,5 & 0 & 0,5 & 0 & 0 & 0 \\
\hline
\end{tabular}

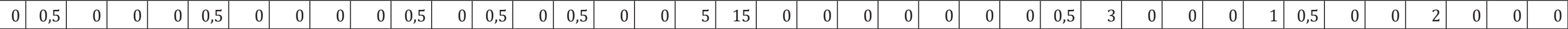

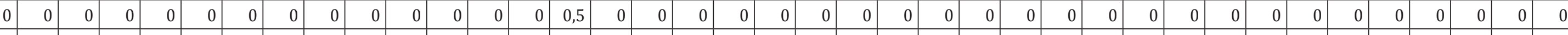

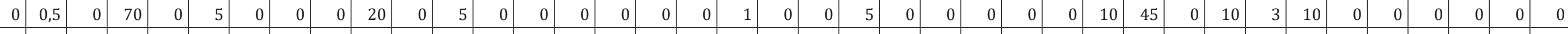

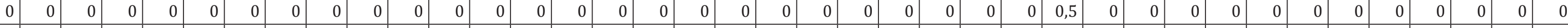

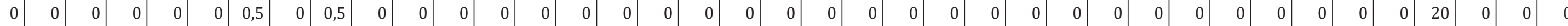

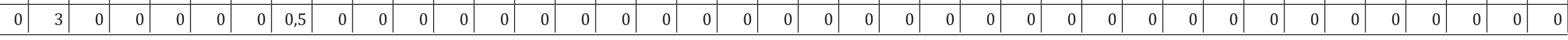




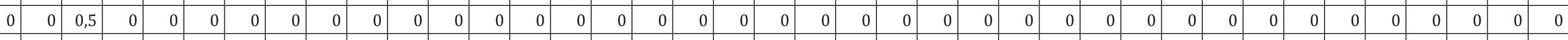
Elymus repens (L.) GOULD

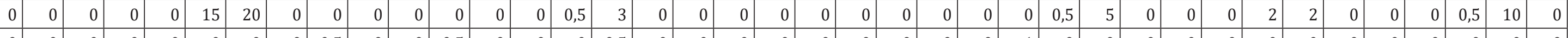

Equisetum arvense $L$.

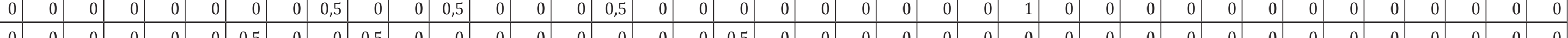

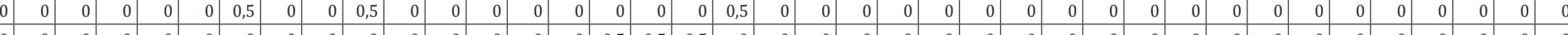

Equisetum sylvaticum $L$.

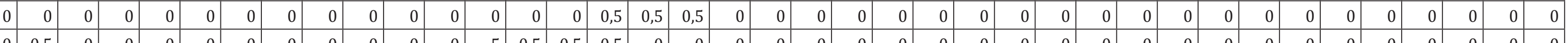

Erigeron annuus (L.) PERS.

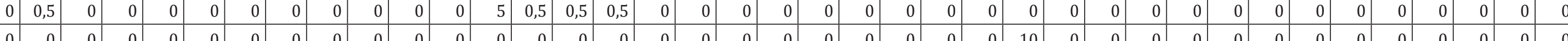
Eupatorium cannabinum $L$

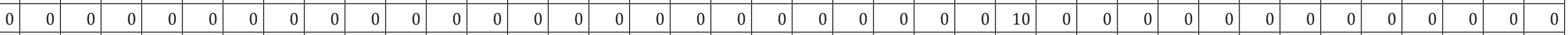

Euphorbia cyparissias $L$. \begin{tabular}{l|l|l|l|l|l|l|l|l|l|l|l|l|l|l|l|l|l|l|l|l|l|l|l|l|l|l|l|l|l|l|l|l|l|l|l|l|l|l}
0 & 0 & 0 & 0 & 0 & 0 & 0 & 0 & 0 & 0 & 0 & 0 & 0 & 0 & 0 & 0,5 & 0,5 & 0 & 0 & 0 & 0 & 0 & 0 & 0 & 0 & 0 & 0 & 0 & 0 & 0 & 0 & 0 & 0 & 0 & 0 & 0 & 0 & 0 & 0 \\
\hline
\end{tabular}

Euphrasia rostkoviana HAYNE

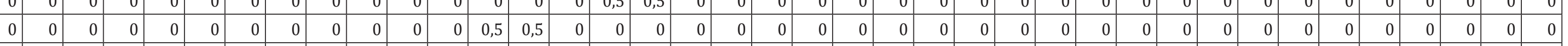
Festuca ovina $L$

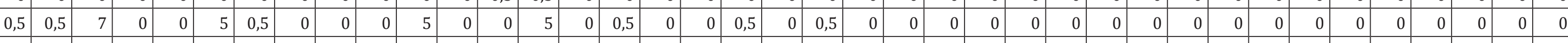
Festuca rubra L. s. S.

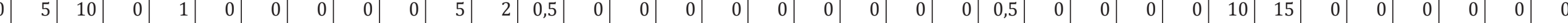

Fragaria viridis DUCHESNE \begin{tabular}{lllllllllllllllllllllllllllllllllllllllllll}
0 & 0 & 0 & 0 & 0 & 0 & 0 & 0 & 0 & 0 & 0 & 0 & 0,5 & 0,5 & 0 & 0 & 0 & 0 & 0 & 0 & 0 & 0 & 0 & 0 & 0 & 0 & 0 & 0 & 0 & 0 & 0 & 0 & 0 & 0 & 0 & 0 & 0 & 0 & 0 \\
\hline
\end{tabular}

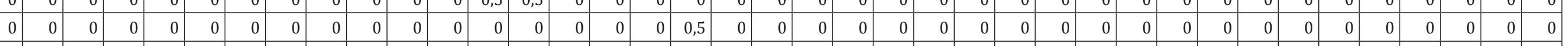

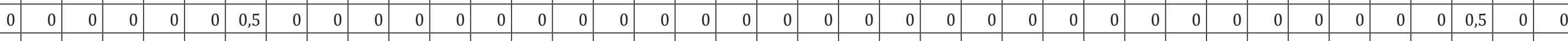

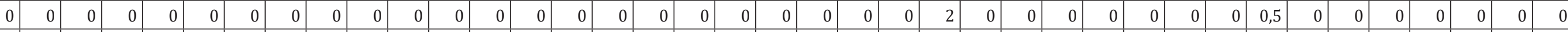

Galeopsis ladanum L. \begin{tabular}{l|lllllllllllllllllllllllllllllllllllllllll}
0 & 0 & 0 & 0 & 0 & 0 & 0 & 0 & 0,5 & 0 & 0 & 0 & 0,5 & 0,5 & 0,5 & 0 & 0 & 0 & 0 & 0 & 0 & 0 & 0 & 0 & 0 & 1 & 0 & 0 & 0 & 0 & 0 & 0 & 0 & 0,5 & 0 & 0,5 & 0 & 0 & 0 \\
\hline
\end{tabular}

Galium mollugo $L$

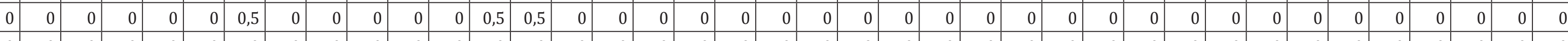

Galium verum $L$.

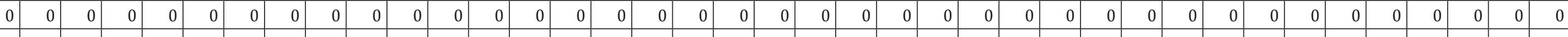
Geum urbanum $L$.

Gnaphalium sylvaticum L

Helichrysum arenarium (L.) MOENCH

Herniaria glabra $L$.

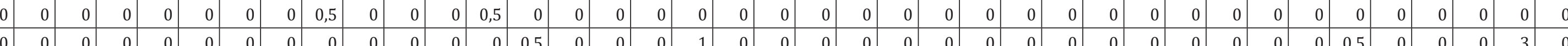
Hieracium pilosella $L$

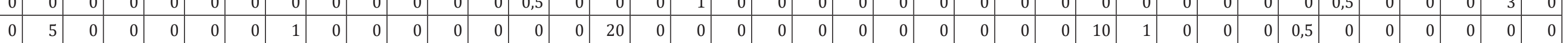

Hieracium umbellatum $L$.

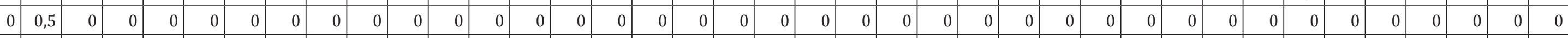

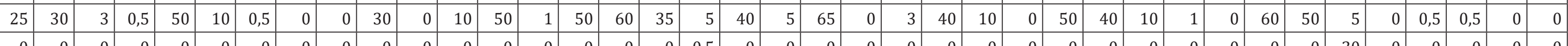

Holcus lanatus $L$.

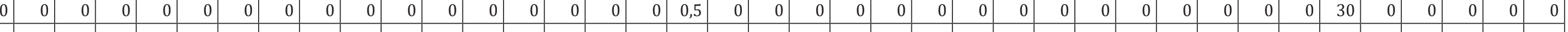
Holcus mollis $L$.

Hypericum perforatum $L$.

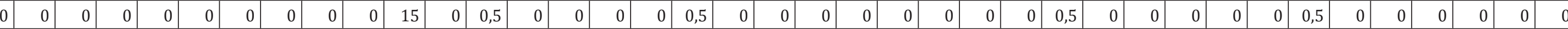
Hypochoeris radicata $L$.

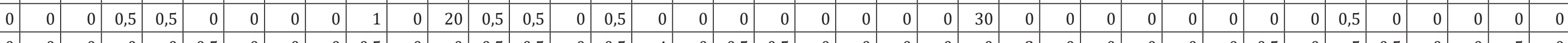

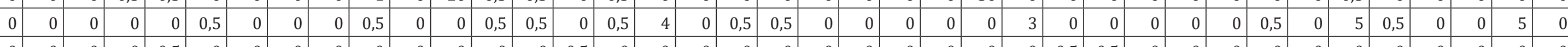

Jasione montana $L$.

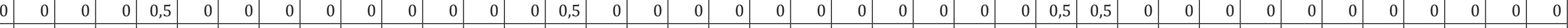

Juncus effusus $L$.

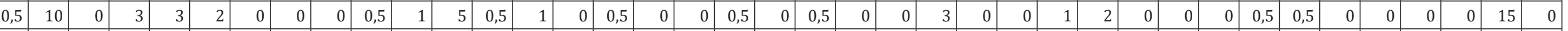

Juniperus communis $L$.

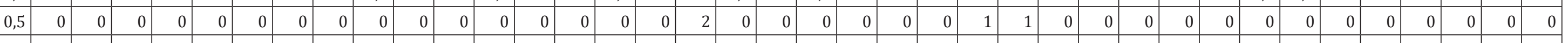
Knautia arvensis (L.) J.M. COULT.

Leontodon autumnalis $L$

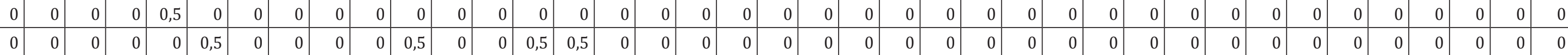

Lupinus polyphyllus LINDL. \begin{tabular}{llllllllllllllllllllllllllllllllllllllllllll}
0 & 0 & 0 & 0 & 0 & 0 & 0 & 0 & 0 & 0 & 0 & 0 & 0 & 0 & 0 & 0 & 0 & 0 & 0 & 0 & 0 & 0 & 0 & 0 & 0 & 0 & 0 & 0 & 0 & 0 & 0 & 0 & 0 & 0 & 0 & 0 & 0 & 0 & 0 \\
\hline
\end{tabular} Luzula campestris (L.) DC.

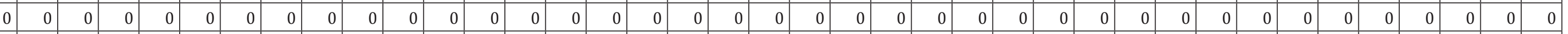

Lysimachia vulgaris $L$

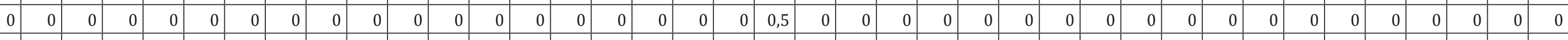
Lythrum salicaria $L$.

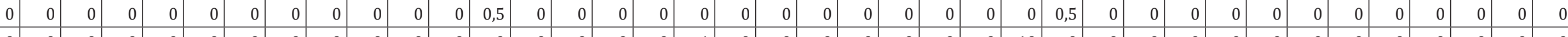

Melandrium album (MHL) GarckE

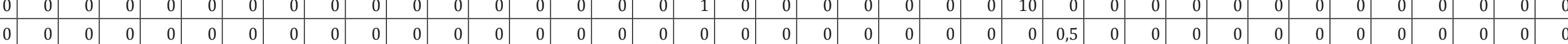


Mentha arvensis $L$.

Mentha $x$ verticillata $L$.

Myosotis arvensis (L.) HILL

Nardus stricta $L$.

Oenothera biennis L. s.s.

Padus serotina (EHRH.) BORKH.

Papaver argemone $L$.

Peucedanum oreoselinum (L.) MOENCH

Phleum pratense $L$.

Pinus sylvestris $L$.

Plantago lanceolata $L$.

Pleurozium schreberi (Brid.) Mitt.

Poa compressa $L$.

Poa pratensis $L$.

Poa trivialis $L$.

Polygonum aviculare $L$.

Polygonym hydropiper $L$.

Polytrichum formosum (Hedw.) G. Sm.

Polytrichum piliferum Hedw.

Potentilla anserina $L$.

Potentilla argentea L. s. $s$.

Pteridium aquilinum (L.) KUHN

Pyrus communis $L$.

Quercus robur $L$

Quercus rubra $L$.

Ranunculus repens $L$.

Rosa sp.

Rubus idaeus $L$.

Rubus caesius $L$.

Rubus sp.

Rumex acetosa $L$.

Rumex acetosella $L$.

Rumex crispus $L$.

Salix caprea $L$.

Scleranthus annuиs $L$.

Danthonia decumbens $D C$

Senecio jacobaea $L$.

Senecio vernalis WALDST. \& KIT.

Senecio viscosus $L$.

Setaria viridis (L.) P. BEAUV.

Solidago virgaurea $L$.

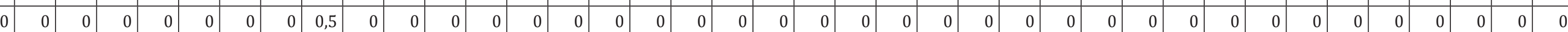

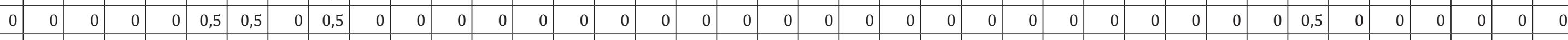

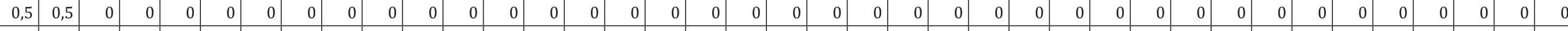
\begin{tabular}{llllllllllllllllllllllllllllllllllllllllllll}
0 & 0 & 0 & 0 & 0 & 0,5 & 0 & 0 & 0 & 0 & 0 & 0 & 0 & 0 & 0 & 0 & 0 & 0 & 2 & 0 & 0 & 0 & 0 & 0 & 0 & 0 & 0 & 0 & 0 & 0 & 0 & 0 & 0 & 0 & 0 & 0 & 0,5 & 0 & 0 \\
\hline
\end{tabular}

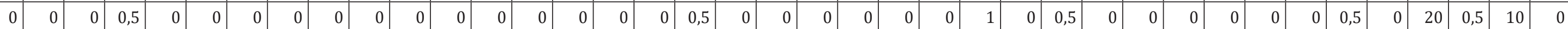

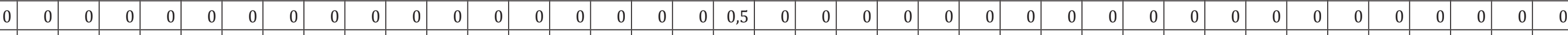

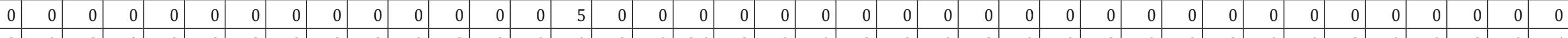
\begin{tabular}{llllllllllllllllllllllllllllllllllllllllll}
0 & 0 & 0 & 0 & 0 & 0 & 0 & 0 & 0 & 0 & 0 & 0 & 0 & 0 & 0 & 0 & 0 & 0,5 & 0 & 0 & 0 & 0 & 0 & 0 & 0 & 0 & 0 & 0 & 0 & 0 & 0 & 0 & 0 & 0 & 0 & 0 & 0 & 0 & 5 \\
\hline
\end{tabular}

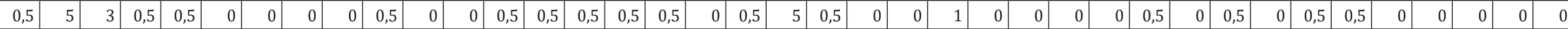

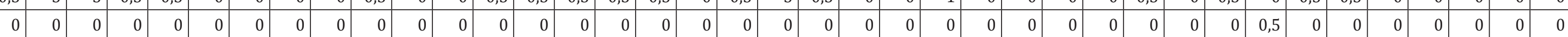

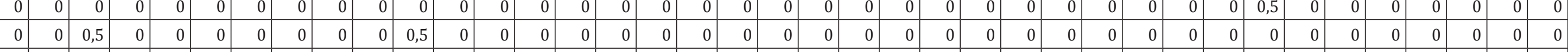

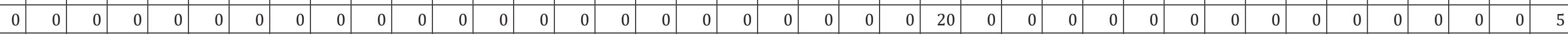

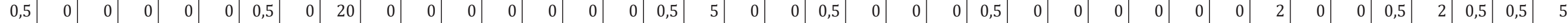

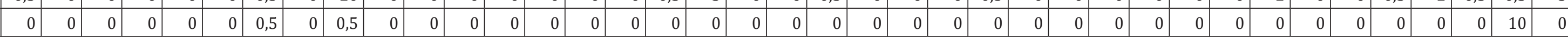

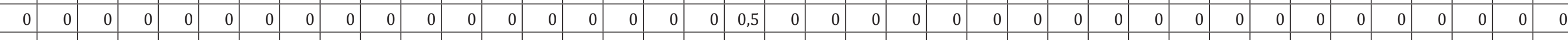

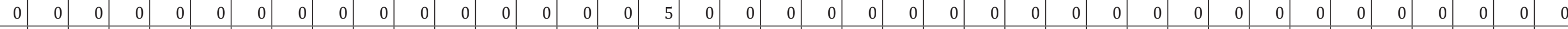

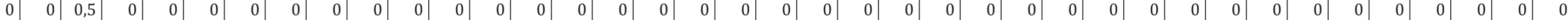
\begin{tabular}{|l|l|l|l|l|l|l|l|l|l|l|l|l|l|l|l|l|l|l|l|l|l|l|l|l|l|l|l|l|l|l|l|l|l|l|l|l|l}
0 & 0 & 0 & 0,5 & 70 & 0 & 0 & 0 & 0 & 1 & 0 & 0,5 & 0 & 0 & 0 & 0 & 0 & 0 & 1 & 0 & 0 & 0 & 0 & 0 & 0 & 0 & 0 & 0 & 0 & 0 & 1 & 0 & 0 & 0 & 0 & 0 & 0 & 0 \\
\hline
\end{tabular}

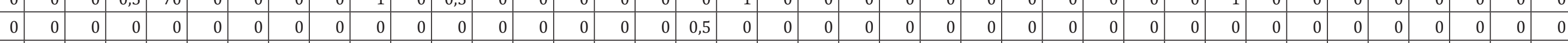

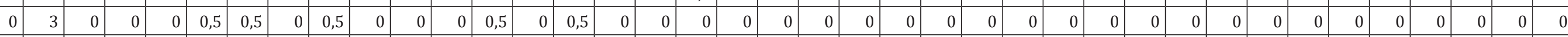
\begin{tabular}{lllllllllllllllllllllllllllllllllllllllllll}
0 & 0 & 0 & 0 & 0 & 0 & 0 & 0 & 0 & 0 & 0 & 0 & 0 & 65 & 40 & 0 & 0 & 0 & 0 & 0 & 0 & 0 & 0 & 0 & 0 & 0 & 0 & 0 & 0 & 0 & 0 & 0 & 0 & 0 & 0 & 0 & 0 & 0 & 0 \\
\hline
\end{tabular}

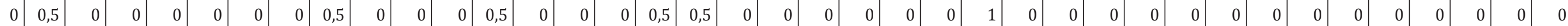

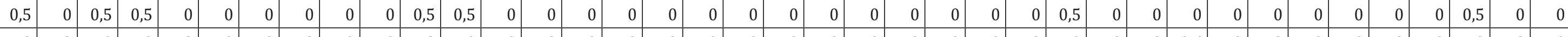

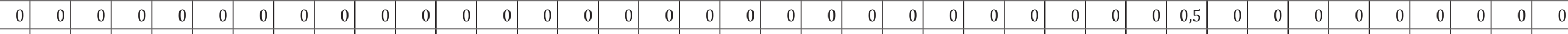
\begin{tabular}{lllllllllllllllllllllllllllllllllllllllllllll}
0 & 0 & 0 & 0 & 0 & 0 & 0 & 0 & 0,5 & 0 & 0 & 0 & 0 & 0 & 0 & 0 & 0 & 0 & 0 & 0 & 0 & 0 & 0 & 0 & 0 & 0 & 0 & 0 & 0 & 0 & 0 & 0 & 0 & 0 & 0 & 0 & 0 & 0 & 0 \\
\hline
\end{tabular}

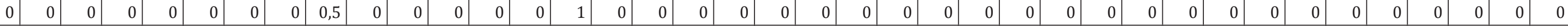

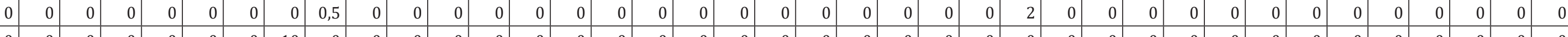

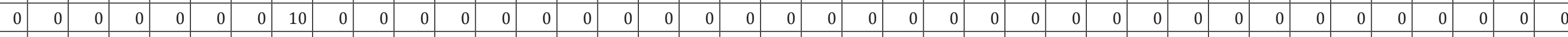

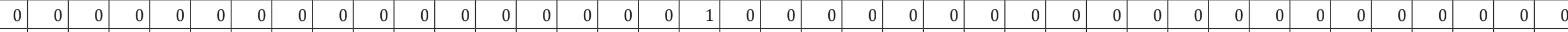

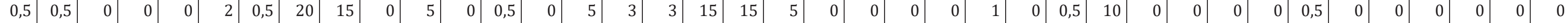

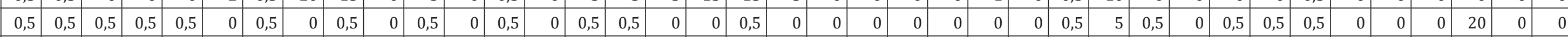

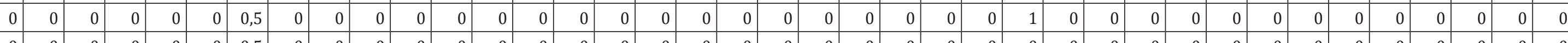

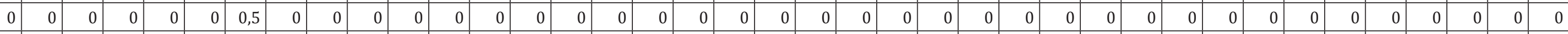
\begin{tabular}{lllllllllllllllllllllllllllllllllllllllllll|l}
0 & 0 & 0 & 0 & 0 & 0 & 0 & 0 & 0 & 0 & 0 & 0 & 0 & 0 & 0 & 0 & 0 & 0 & 0,5 & 0 & 0 & 0 & 0 & 0 & 0 & 0 & 0 & 0 & 0 & 0 & 0 & 0 & 0 & 0 & 0 & 0 & 0 & 0 & 0 \\
\hline
\end{tabular}

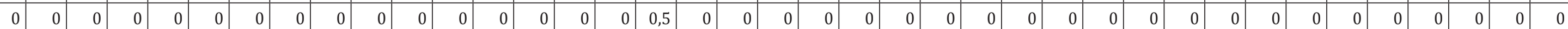

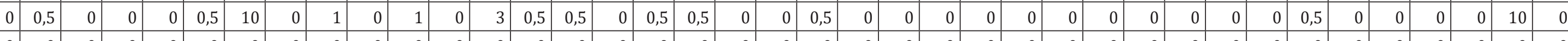

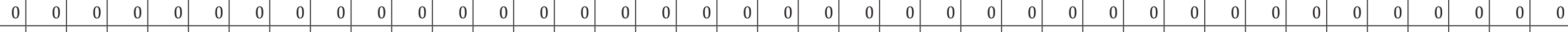
\begin{tabular}{llllllllllllllllllllllllllllllllllllllllllllllll}
0 & 0 & 0 & 0 & 0 & 0 & 0 & 0 & 0 & 0 & 0 & 0 & 0 & 0 & 0 & 0 & 0 & 0 & 0 & 0 & 0 & 0 & 0 & 0 & 0 & 0 & 0 & 0 & 0 & 0 & 0 & 0 & 0 & 0 & 0 & 30 & 0 & 0 & 0 \\
\hline
\end{tabular}

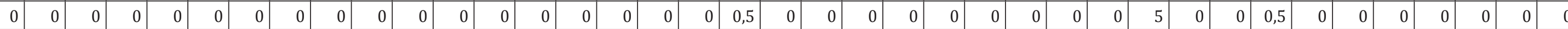

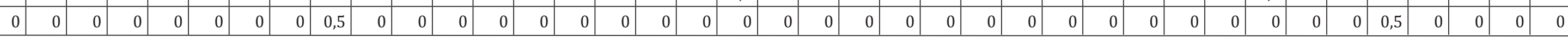


Solidago canadensis $L$.

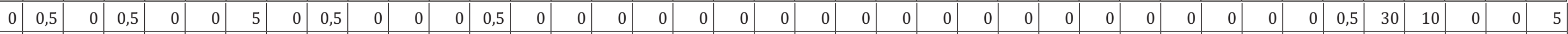
Sonchus arvensis $L$.

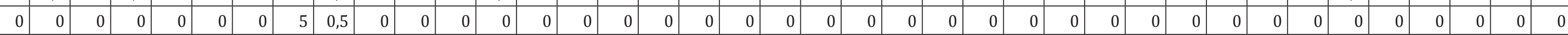

Sorbus aucuparia L. em. HEDL.

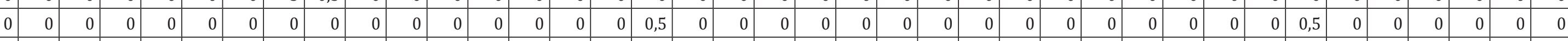

Spergula morisonii BOREAU \begin{tabular}{|lllllllllllllllllllllllllllllllllllllllllll}
0 & 0 & 0 & 0 & 0 & 0 & 0 & 0 & 0 & 0 & 0 & 0 & 0 & 0 & 0 & 0 & 0 & 0 & 0 & 0 & 0 & 0 & 0 & 0 & 0 & 0 & 0 & 0 & 0 & 0 & 0 & 0 & 0 & 0 & 0,5 & 0 & 0 \\
\hline
\end{tabular}

Spergularia rubra (L.) J. PRESL \& C. PRESL \begin{tabular}{lllllllllllllllllllllllllllllllllllllllllll}
0 & 0 & 0 & 0 & 0 & 0 & 0 & 0 & 0 & 0,5 & 0 & 0 & 0 & 0 & 0 & 0 & 0 & 0 & 0 & 0 & 0 & 0 & 2 & 0 & 0 & 0 & 0 & 0 & 0 & 0 & 0 & 0 & 0 & 0 & 0 & 0 & 0 & 0 & 0 \\
\hline
\end{tabular}

Stachys recta L.

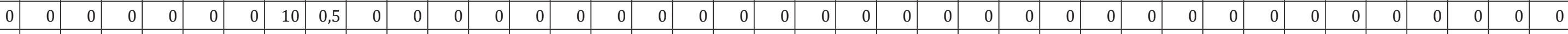

Stellaria graminea $L$.

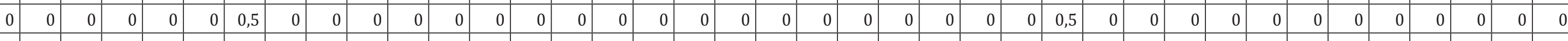

Tanacetum vulgare $L$

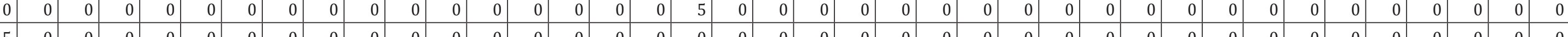

Taraxacum officinale F. H. WIGG

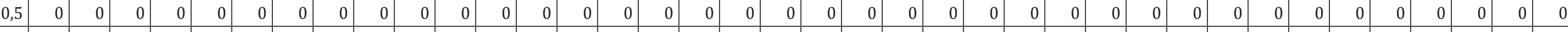

Thymus pulegioides

\begin{tabular}{lllllllllllllllllllllllllllllllllllllllllll}
0 & 0 & 0 & 0 & 0 & 0 & 0 & 0 & 0 & 0 & 0 & 0 & 0 & 0 & 0 & 0 & 0 & 0 & 0 & 0 & 0 & 0 & 0 & 0 & 0 & 0 & 0 & 0 & 0 & 0 & 0 & 0 & 0 & 10 & 0 & 0 & 0 & 0 & 0 \\
\hline
\end{tabular}

Tragopogon pratensis L. s. s.

Trifolium arvense $L$.

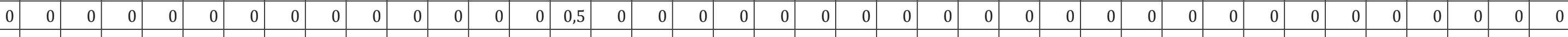

Trifolium repens $L$

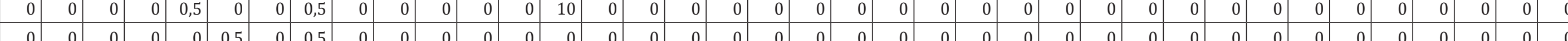

Urtica dioica L

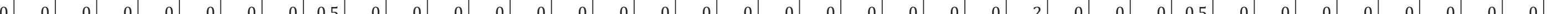

Veronica chamedrys $L$.

Veronica officinalis $L$

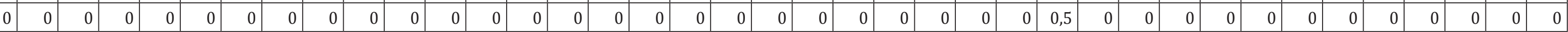
Vicia angustifolia $L$.

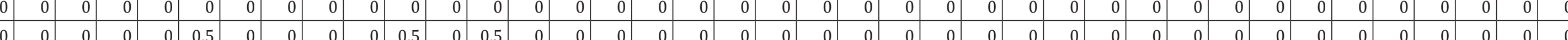

Vicia cracca L.

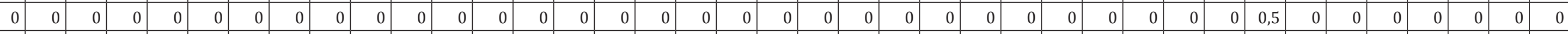

Vicia hirsuta (L.) S. F. GRAY

Vicia tenuifolia ROTH

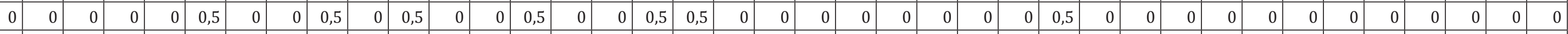

Vicia tetrasperma (L) SCHREB.

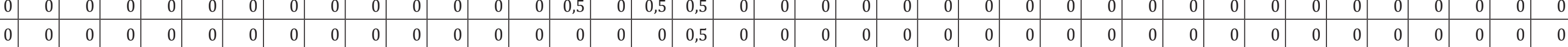

Viola arvensis MURRAY

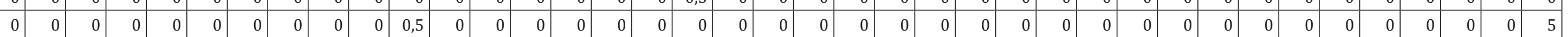

Viola reichenbachiana JORD. ex BOREAU

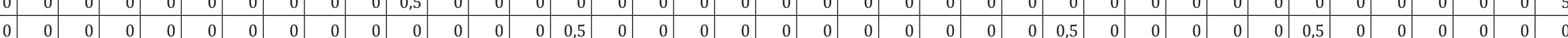
Viola tricolor L. s. S.

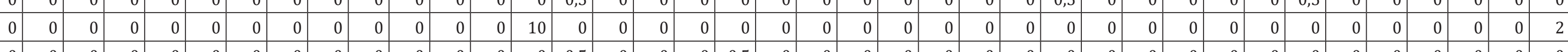

Source: own elaboration. 
The most uniform was cluster $\mathrm{Z}$, which grouped study plots representing the same community type, with Agrostis capillaris, Hieracium pilosella and Achillea millefolium. Less uniform is cluster $\mathrm{X}$, which groups patches of a poor community with Anthoxanthum aristatum and Corynephorus canescens and patches similar to the more diverse community with Agrostis capillaris, Hieracium pilosella and Achillea millefolium.
The most heterogeneous is cluster Y. It groups patches of the floristically poor community with Calamgrostis epigejos; patches of a community rich in plant species, with Cirsium arvense, Galium mollugo and Gnaphalium sylcaticum; the few patches of phytocoenoses with Cirsium arvense and Solidago canadensis; a community of grasses and perennial plants with Betula pendula and a community with lichens of the Cladonia genus.

Table 6.3. Relative cover (P), relative frequency (F) and IndVal indicator value (I) (all in \%) in individual clusters (for species associated with any cluster at $\mathrm{p}<0.1$ ).

The significantly (at $\mathrm{p} \leq 0.05$ ) highest IndVals are in bold

\begin{tabular}{|c|c|c|c|c|c|c|c|c|c|c|c|c|c|c|c|c|c|}
\hline \multicolumn{2}{|c|}{ Cluster } & 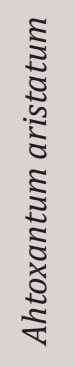 & 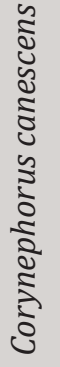 & $\begin{array}{l}\text { के } \\
\text { है } \\
\text { ते } \\
\text { के }\end{array}$ & 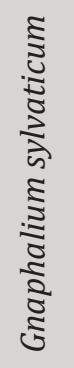 & 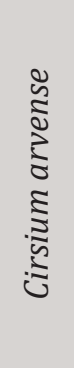 & 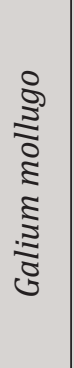 & 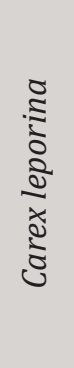 & 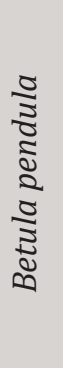 & 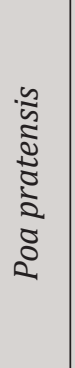 & 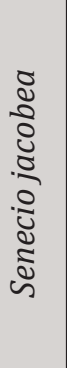 & 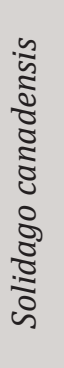 & 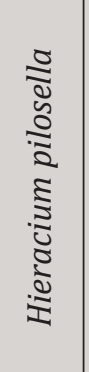 & 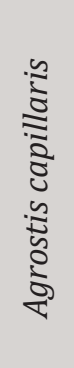 & 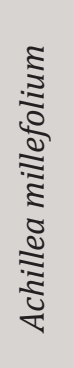 & 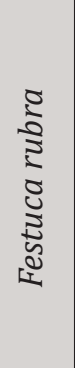 & 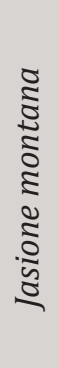 \\
\hline \multicolumn{2}{|c|}{$p$} & $\begin{array}{l}\stackrel{D}{\mathscr{N}} \\
\stackrel{0}{0} \\
\dot{0}\end{array}$ & $\begin{array}{l}\text { L } \\
\text { م⿱ } \\
0 \\
0 \\
0\end{array}$ & $\begin{array}{l}\stackrel{N}{\infty} \\
\stackrel{+}{+} \\
\dot{0}\end{array}$ & $\begin{array}{l}\infty \\
\stackrel{8}{0} \\
\stackrel{0}{0} \\
\dot{0}\end{array}$ & $\begin{array}{l}\text { मे } \\
\text { } \\
\dot{0} \\
\dot{0}\end{array}$ & $\begin{array}{l}\vec{H} \\
0 \\
- \\
0 \\
0\end{array}$ & $\begin{array}{l}0 \\
0 \\
0 \\
0 \\
0\end{array}$ & $\begin{array}{l}\stackrel{+}{m} \\
\text { On } \\
\stackrel{0}{0}\end{array}$ & $\begin{array}{l}0 \\
\mathscr{2} \\
\infty \\
0 \\
0\end{array}$ & \begin{tabular}{l} 
L \\
\multirow{2}{*}{} \\
0 \\
0 \\
0
\end{tabular} & $\begin{array}{l}{ }^{+} \\
8 \\
0 \\
0\end{array}$ & $\begin{array}{l}\overrightarrow{-} \\
\stackrel{-}{8} \\
\stackrel{0}{0}\end{array}$ & \begin{tabular}{l}
0 \\
\multirow{2}{0}{} \\
0 \\
0
\end{tabular} & $\begin{array}{l}\infty \\
\stackrel{0}{0} \\
\stackrel{1}{0} \\
\stackrel{0}{0}\end{array}$ & $\begin{array}{l}0 \\
0 \\
\infty \\
0 \\
0 \\
0\end{array}$ & $\begin{array}{l}N \\
\infty \\
o \\
0 \\
0 \\
0\end{array}$ \\
\hline \multirow{3}{*}{$X$} & $\mathrm{P}$ & 100 & 87 & 100 & 0 & 0 & 0 & 0 & 0 & 0 & 0 & 1 & 31 & 0 & 7 & 4 & 20 \\
\hline & $\mathrm{F}$ & 42 & 58 & 25 & 0 & 0 & 0 & 0 & 0 & 0 & 0 & 8 & 75 & 17 & 25 & 8 & 50 \\
\hline & I & 42 & 51 & 25 & 0 & 0 & 0 & 0 & 0 & 0 & 0 & 0 & 23 & 0 & 2 & 0 & 10 \\
\hline & & & & & & & & & & & & & & & & & \\
\hline \multirow{3}{*}{$\mathrm{Y}$} & $P$ & 0 & 0 & 0 & 100 & 100 & 85 & 100 & 94 & 85 & 77 & 96 & 7 & 42 & 44 & 28 & 29 \\
\hline & $\mathrm{F}$ & 0 & 0 & 0 & 33 & 40 & 40 & 40 & 47 & 47 & 47 & 33 & 60 & 60 & 40 & 20 & 20 \\
\hline & I & 0 & 0 & 0 & 33 & 40 & 34 & 40 & 44 & 40 & 36 & 32 & 4 & 25 & 17 & 6 & 6 \\
\hline & & & & & & & & & & & & & & & & & \\
\hline \multirow{3}{*}{$\mathrm{Z}$} & $P$ & 0 & 13 & 0 & 0 & 0 & 15 & 0 & 6 & 15 & 23 & 3 & 61 & 57 & 49 & 68 & 50 \\
\hline & $\mathrm{F}$ & 0 & 42 & 0 & 0 & 0 & 8 & 8 & 25 & 42 & 50 & 17 & 100 & 100 & 92 & 42 & 83 \\
\hline & I & 0 & 5 & 0 & 0 & 0 & 1 & 0 & 2 & 6 & 11 & 0 & 61 & 57 & 45 & 28 & 42 \\
\hline
\end{tabular}

Source: own elaboration.

\section{Community with Anthoxanthum aristatum and Corynephorus canescens}

It is a floristically poor community of grasses. The highest IndVal value was obtained by two species here: Anthoxanthum aristatum and Corynephorus canescens (cluster X). Some study plots were overgrown only with Corynephorus canescens (e.g. Celestynów A). The two species dominate as regards land cover and shape the physiognomy of this community. The share of other species is in- significant (Tab. 6.1). The most typical patches include: Celestynów A, Krzętle A (Photo 6.4), Wola Makowska A, Wola Makowska B, Polesie C. The physiognomically similar patches of study plots Polesie B and Polesie C, which are very poor floristically, and their dominating species are grasses Anthoxanthum aristatum or Poa compressa, can also be included in this community. 


\section{Community with Agrostis capillaris, Hieracium pilosella and Achillea millefolium}

These are floristically poor phytocoenoses, in which the species with significant values of IndVal were: Agrostis capillaris, Hieracium pilosella and Achillea millefolium (cluster Z). All patches of phytocoenoses that are included in cluster $\mathrm{Z}$ constitute the typical form of this community (Fig. 6.1). Only study plots Piskorzeniec $\mathrm{C}$ and Wola Życińska A are characterised by a high share of Holcus mollis, higher than that of Hieracium pilosella. In some plots, there are small numbers of tree saplings (Betula pendula, Pinus sylvestris).

This community is represented by study plots Wola Pszczółecka A, Weronika A and Piskorzeniec
C, included in cluster X. These patches are richer in plant species, with a large share of Hieracium pilosella. They probably constitute a transitory link from poor communities of grasses with a small share of perennial plants, to the floristically richer phytocoenoses of meadow character. Study plots Glinnik C, Celestynów C and Krzętle $\mathrm{B}$, included in cluster $\mathrm{Y}$, are also physiognomically similar to this community, because their dominating species is Agrostis capillaris, accompanied by a significant share of Hieracium pilosella or Elymus repens.

\section{Community with Calamgrostis epigejos}

The physiognomy of this community is shaped by dense occurrence of Calamgrostis epigejos, assisted by a small addition of Agrostis capillaris, Hieracium pilosella, Eupatorium cannabinum, Cirsium arvense and Equisetum arvense. Among the analysed abandoned lands, only three patches dominated by Calamgrostis epigejos were found. They were: Wola Życińska B, Wola Makowska C and
Szymaniszki A (Tab. 6.1). The study plots were included in cluster Y (Fig. 6.3). The least typical is plot Szymaniszki A, which differs from the others by the much greater number of species (17), a smaller share of Calamgrostis epigejos and a high share of perennial plants, such as Hieracium umbellatum.

\section{Community with Cladonia}

Patches of this phytocoenosis are characterised by a very small share of vascular plants, mainly: Corynephorus canescens and Arnoseris minima. The plants cover an insignificant percentage of the plot. The patches are dominated by lichens of the Cladonia genus, mostly Cladonia arbuscula and $C l$. rangiferina. They are accompanied by Cladonia strepsillis f. coralloides (Photo 7.6), Cl. uncialis and $\mathrm{Cl}$. furcata. Patches of this community were recorded at study plots: Piskorzeniec A, Polesie A and Wola Pszczółecka B, included in cluster X.

\section{Community with Cirsium arvense, Galium mollugo and Gnaphalium sylcaticum}

It is a floristically rich community, with multi-species flora (Tab. 6.1). A significant value of IndVal was reached by 5 species: Cirsium arvense, Galium mollugo, Gnaphalium sylcaticum and Betula pendula. This phytocoenosis is represented by two study plots: Raciszyn C and Weronika C (cluster Y). Seve- ral meadow species were identified here, such as Galium mollugo, Stellaria graminea, Viccia cracca, Trifolium repens; species of xerothermic grasslands, e.g. Dianthus carthusiana, and a few forest species, e.g. Gnaphalium sylcaticum.

\section{Community with Elymus repens or Poa pratensis}

This phytocoenosis is dominated by grasses - Elymus repens or Poa pratensis. In patches of this community, a high share of Senecio jacobea or Rumex acetosa is also noticeable. The community is very abundant floristically (Tab.6.1). Fo- rest species, meadow species, and species of dry grasslands occur here. Typical patches of this community - Sulejów A and Sulejów C were included in cluster Y. 


\section{Community with Cirsium arvense and Solidago canadensis}

The phytocoenosis is represented in the analysed area by only one study plot - Szymaniszki B, included in cluster Y. Floristically, it is a very poor community. Two species dominate here: Cirsium arvense and Solidago canadensis. The share of three other species is insignificant (Tab. 6.1).

\section{Community with Betula pendula}

This type of community was identified at two study plots only: Łagiewniki B and Łagiewniki $\mathrm{C}$, included in cluster $\mathrm{Y}$. The physiognomy of this phytocoenosis is shaped by a considerable share of Betula pendula. There are not many herba- ceous plants there (8-12). The dominating ones are: Elymus repens, Conyza canadensis, Senecio jacobea, Jasione montana and Rumex acetosa (Tab. 6.1).

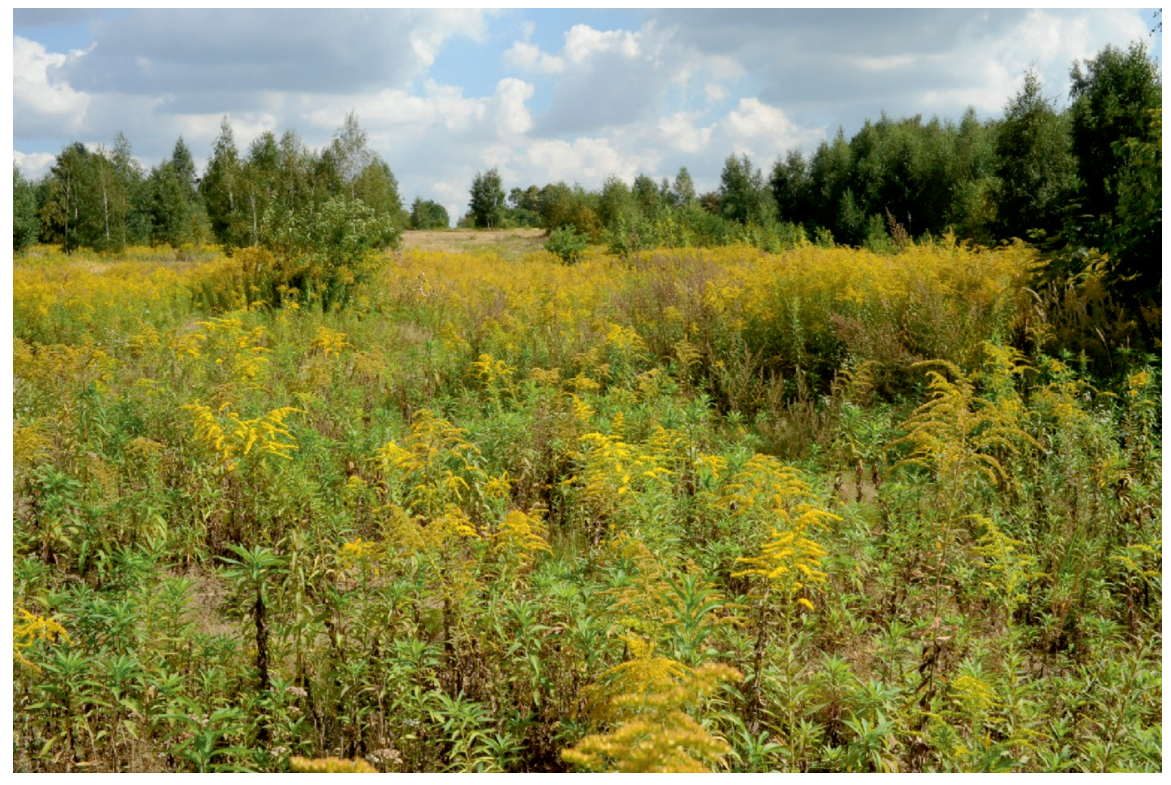

Photo 6.1. Solidago canadensis in Sulejów A (S. Krysiak)

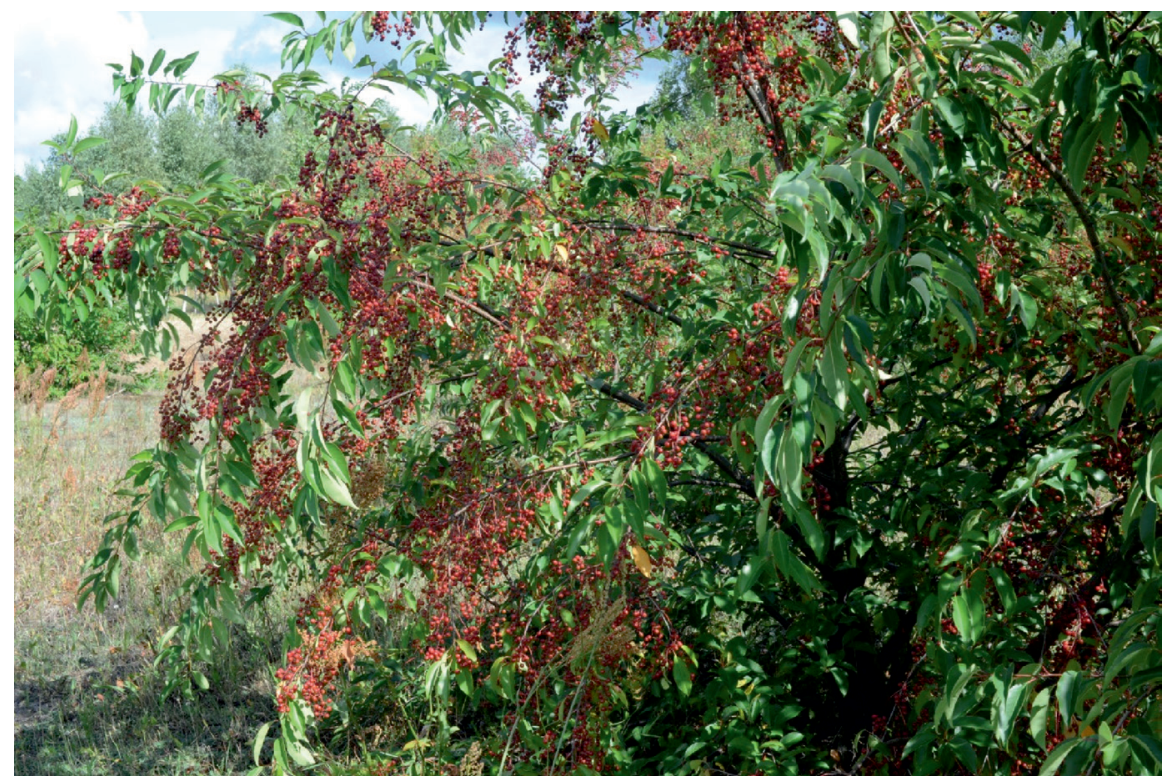

Photo 6.2. Padus serotina in Wola Życińska A (S. Krysiak) 


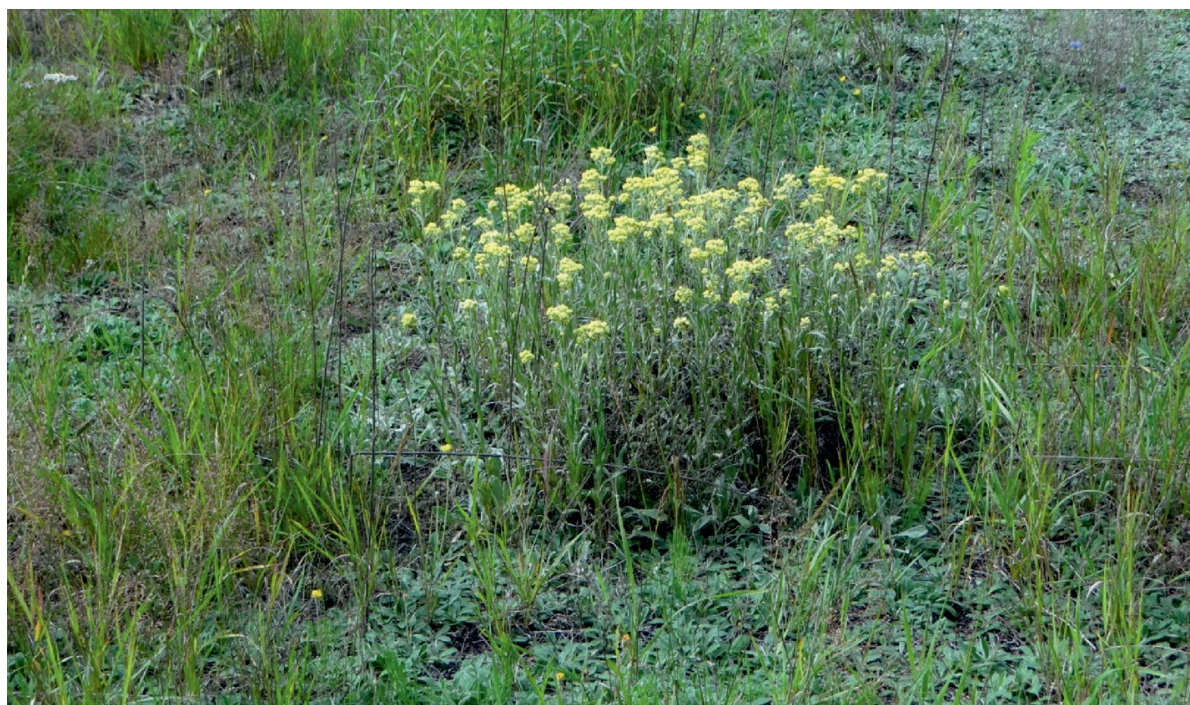

Photo 6.3. Helichrysum arenaria in Weronika A (E. Papińska)

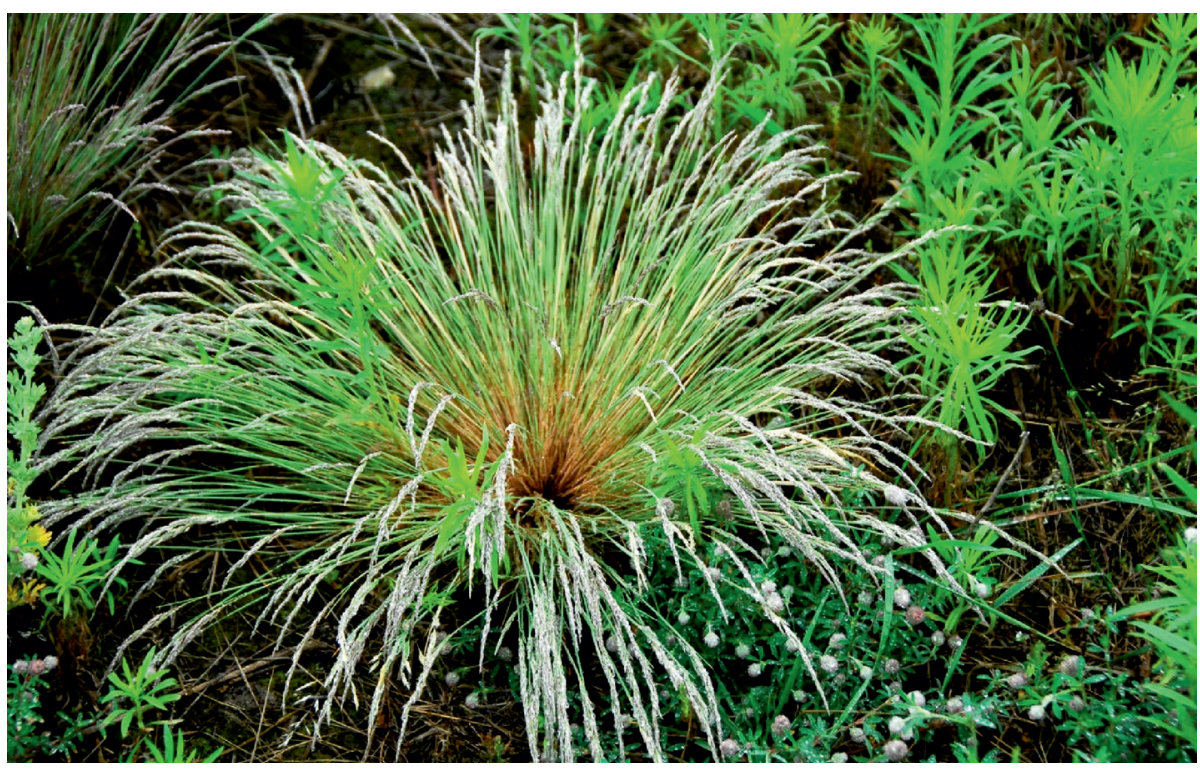

Photo 6.4. Corynephorus canescens in Krzętle A (E. Papińska)

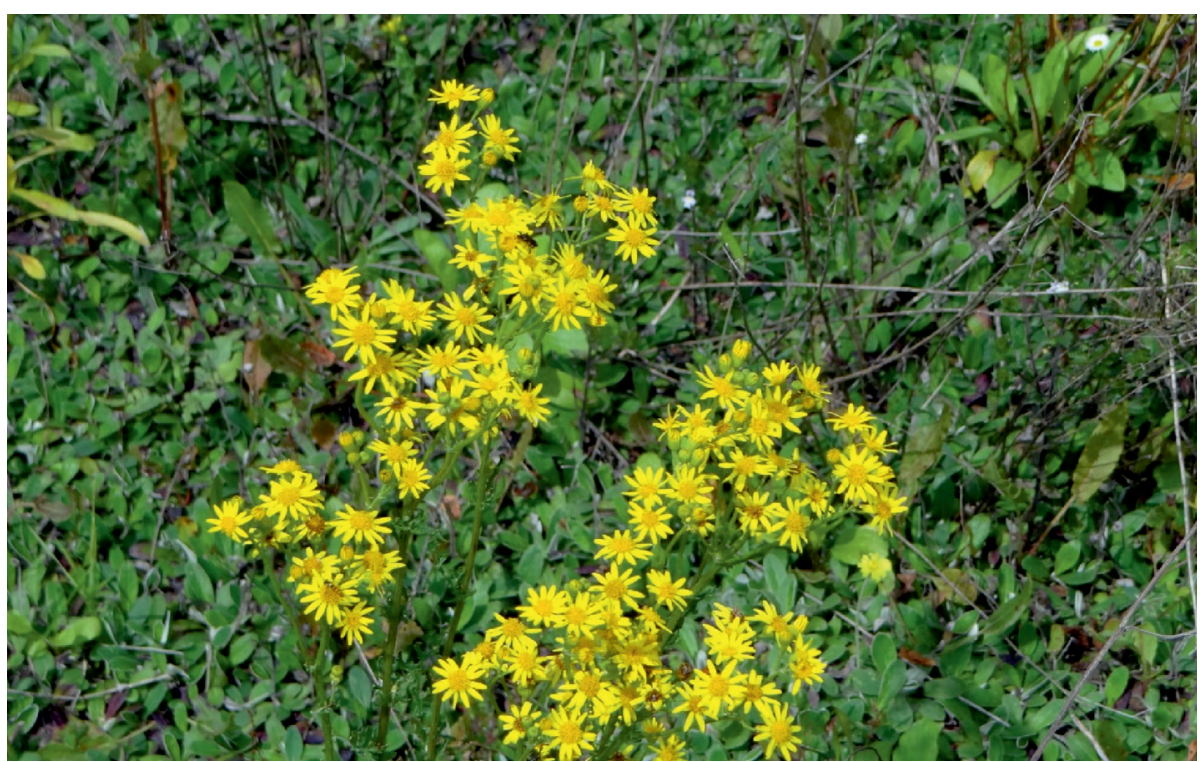

Photo 6.5. Senecio jacobaea in Raciszyn A (E. Papińska) 


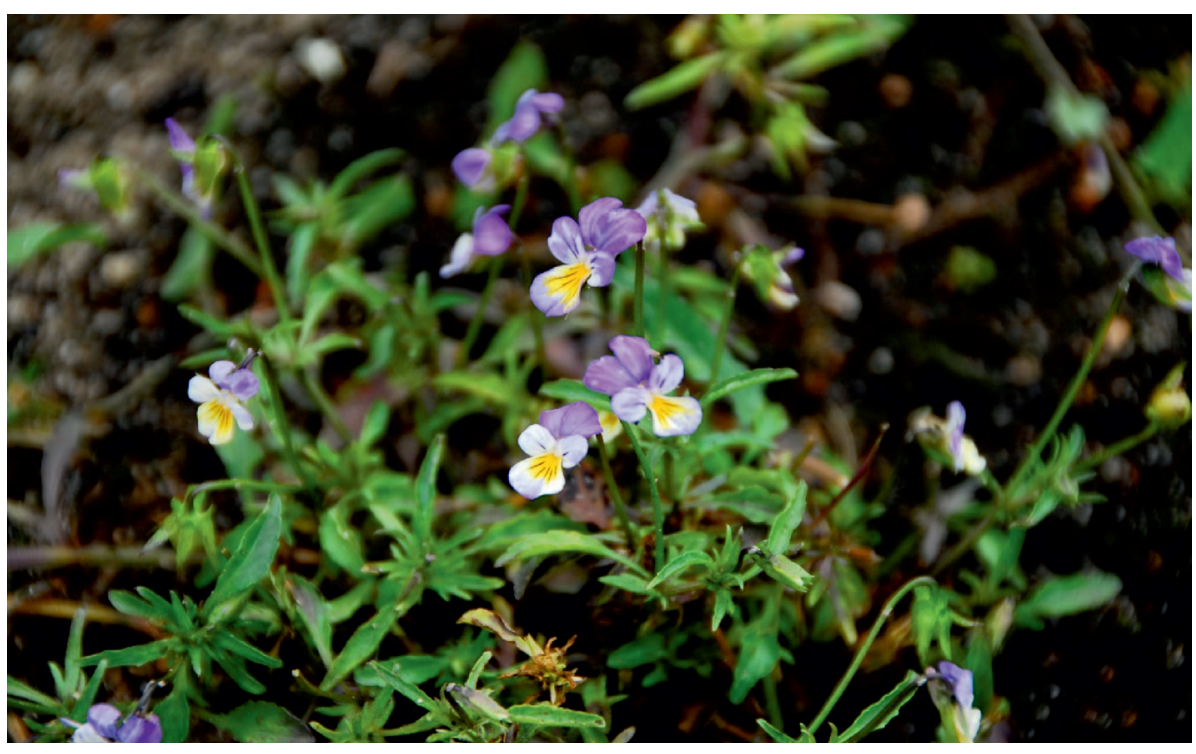

Photo 6.6. Viola tricolor in Raciszyn C (E. Papińska) 